\title{
Haralick Texture Features Based on Bag of Visual Words for a Spine MRI Images
}

\author{
${ }^{1}$ Khawlah H. Ali, ${ }^{2}$ Entesar B. Talal
}

\begin{abstract}
- this paper explores statistical features of texture based image descriptors that make use of the spatial gray level of bag of visual words model to efficiently improve classification performance for two types of spine MRI images, which is normal and abnormal(which is may be a cancer). At first, texture is characterized through second order statistical measurements based on the gray-level co-occurrence matrix introduced by Haralick. By this method it is possible to compute, four features which are designed to perform texture: contrast, correlation, homogeneity, and energy for spine MRI images, and then construct a bag of visual word (BoW) to encode feature vector into visual words. Features of these types are used to classify two categories of spine MRI image: normal and abnormal, then, classify them by using SVM, which is works efficiently. Experiment results on spine MRI show significant improvement of classification.
\end{abstract}

Keywords :gray level co-occurrence matrix, visual words, K-means, classification

\section{Introduction}

In recent years, there are developments in digital images especially in medical images in various modes, such as X-rays, magnetic resonance imaging (MRI), computer tomography (CT), ultrasonography (US), etc. [14].

Medical image classification is an important issue for physicians in diagnoses for Clinical and medical researches. However, traditional methods of medical image classifications based on global features include color features, texture features and shapes features have many inaccurate classifications [14].

Computer Dept., College of Education, Univ.of Basrah Iraq

Computer Dept., College of Education, Univ.of Basrah Iraq
Medical image classification has been grow and active research in biomedicine field. In this paper we deal with MRI scan of spinal structures to help the diagnose what cause back-pain.

In recent years, with the massive development of local descriptors in computer vision and pattern recognition, the bag of visual words extracting from local features like key points or image patches has introduced as activated search for image classification and image retrieval $[1,2,12,13$ and 14$]$.

Image classifications have been significantly active topic research with hundreds of publications in the past years. Texture features are often used to analyze images especially for medical images. In this paper, at first, texture features are analyzed by using gray-level co-occurrence matrix then create bag of visual words, usually K-means are used to cluster centers of features which are extracted from all training images, then these cluster centers are used to generate visual word vocabulary for all images to get word vector representations.

In [6] introduced an X-ray image categorization and retrieval method based on batches of visual words. In [3] improve SIFT features for medical images, while in [5] evaluate different representations of histopathology by using SIFT and bag of visual words. All the above methods construct the histogram for image representation by assigning the descriptors of local features to a single visual word has nearest in the vocabulary, which is called nearest neighbor (NN) assignment [14]. The medical applications in [4, 5 and 6] do not investigate of newest development of BoW like sparse coding or development of histogram of visual words in their applications. In this paper, we try to develop classification of medical images by combine co-occurrence matrix with bag of visual words as shown in Fig.1. 


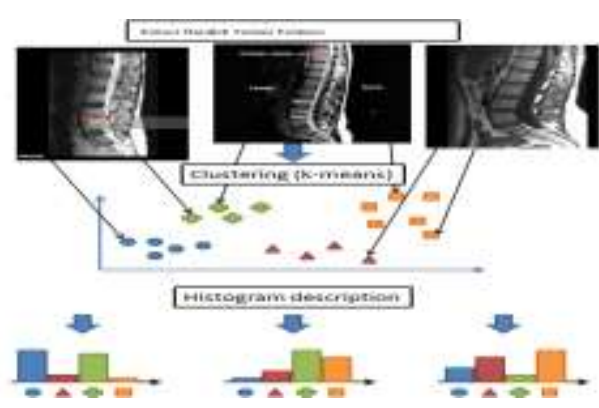

Figure 1. Creation of vocabulary visual words.

\section{Texture features for medical images}

Texture is described as normal property in all most surfaces. The complex nature of texture features has derived a large number of representations. In the perceptual type of texture features consist of features that are corresponding to visual human interpretation. In this paper, we used second order of statistical measurements through gray level co-occurrence matrix (GLCM).Their importance due to their properties with the human perception. The set of perceptual texture feature that Haralick et al. (1973) presented were: contrast, correlation, homogeneity and Energy. We used these feature for MRI spine images as show in table I. A common problem for the spine surgeons is that the mixed messages. A loss of water content in a disc makes it look darker than others, this can be normal activity [14]. In 1973, Haralick [9] introduced 14 statistical features. These features are generated by calculating the features for each one of the co-occurrence matrices obtained by using the directions $0^{\circ}, 45^{\circ}, 90^{\circ}$ and $135^{\circ}$ cover the orientation [1], as shown in Fig. 2, then averaging these four values, and the most common choice of distance is $d=1$ when $\theta$ is $0^{\circ}$ or $90^{\circ}$, and $d=$ $\sqrt{2}$ when $\theta$ is $45^{\circ}$ or 135 . Mathematically, for a given image $I$ of size $K \times K$, the elements of a graylevel Co-occurrence matrix [1] as shown in Fig. 1 for a displacement vector $(\mathrm{d}=d x, d y)$ is defined as:

$$
M_{c o}(i, j)=\left\{\begin{array}{l}
1 \text { if }(x, y)=i \text { and } I\left(x+d_{x}, y+d_{y}\right)=j \\
0 \text { otherwise }
\end{array}\right.
$$

A unique GLCM might not be enough to describe the textural features of the medical image [1]. To create multiple GLCMs, specify an array of offsets. These offsets define pixel relationships of varying direction and distance. The distance parameter can be selected as one or higher. A vector of these 14 statistical features is used for characterizing the cooccurrence matrix contents [1], only four of them are defined here:

- The Contrast: Measures the local contrast of an image. The Contrast is expected to be low if the gray levels of each pixel pair are similar, given by $[1,2]$ :

$$
\text { Contrast }=\sum_{i=1}^{M} \sum_{j=1}^{N}(i-j)^{2} P(i, j)
$$

- The Correlation: Supplies a correlation between the two pixels in the pixel pair. The Correlation is depended to be high if the gray levels of the pixel pairs are highly correlated.

$$
\text { Correlation }=\sum_{i=1}^{M} \sum_{j=1}^{N} \frac{(1-\mu)(j-\mu) P(i, j)}{\sigma^{2}}
$$

- The Homogeneity: Measures the local homogeneity of a pixel pair. The Homogeneity is expected to be large if the gray levels of each pixel pair are similar given by:

$$
\text { Homogeneity }=\sum_{i=1}^{M} \sum_{j=1}^{N} \frac{P[i, j]}{1+|i-j|}
$$

- The Energy: Measures the number of repeated pairs. The Energy is expected to be high if the occurrence of repeated pixel pairs is high, given by:

$$
\text { Energy }=\quad \sum_{i=1}^{M} \sum_{j=1}^{N} P^{2}(i, j)
$$

Where $\mathrm{M}, \mathrm{N}$ are the dimensions of image $\mathrm{P}(\mathrm{i}, \mathrm{j})$. $\sigma$ and $\mu$ are the standard deviation and mean of image displacement respectively $[1,8,9]$.

With large numbers of texture features and for best representation, it is natural to combine each feature value to one feature vector. In next section we used bag of visual words for these texture features.

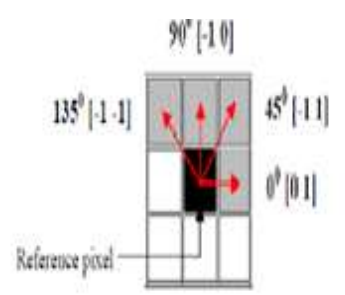

\begin{tabular}{|c|c|c|c|c|}
\hline Feature & normal & Value 1 & Abnormal & Value2 \\
\hline Contrast & & 1.5056 & & 0.1003 \\
\hline $\begin{array}{l}\text { Correlati } \\
\text { on }\end{array}$ & & 2.9617 & & 2.4015 \\
\hline Energy & & 0.8210 & & 2.9666 \\
\hline
\end{tabular}

Figure 2.Co-Occurrence Matrices For Four Directions TABLE I

CO-OCCURRENCE MATRIX FEATURES OF SPINE MRI IMAGES 


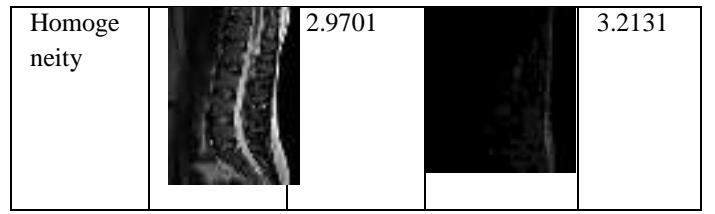

III. Bag of visual features based texture features for medical images

The basic steps of the bag of visual words based classification on texture features are illustrated in Fig.1, where each component represents an operation of the images. To extract local features, we represent an input image as a collection of local features [7].

The first step is detected local features. Let $\mathrm{X}$ be a bag of features of an image, and $\left\{\mathrm{x}_{1}\right\}$, $\mathrm{L}=1, \ldots \ldots \mathrm{L}$ is a collection of local features extracted from X. practically, local features $\mathrm{x}_{1}$ are detected texture features with Haralick descriptors. The second step is constructing visual vocabulary words by using K-means clustering, which cluster the center of features which are extracted from all images. The last step is assigning the descriptors to visual words, the image $\mathrm{X}$ is coded by the local feature $\left\{\left(\mathrm{x}_{1}, \alpha_{1}\right)\right\}, \mathrm{L}=1, \ldots . \mathrm{L}$, with each $\alpha_{\mathrm{k}}$ identified with the integers $\mathrm{k}=1, \ldots \mathrm{K},[7]$.

$$
\alpha_{1}=\arg \min _{\mathrm{k}}\left(\mathrm{D}\left(\mathrm{V}_{\mathrm{k}}, \chi_{\mathrm{l}}\right)\right)
$$

Where $\chi_{l}$ is an image region $\mathrm{L}$, and $\left.\mathrm{D}\left(\mathrm{V}_{\mathrm{k}}, \chi_{\mathrm{l}}\right)\right)$ is the distance between a code word $v_{k}$ and region $\chi_{l}$ [7]. The histogram of visual words is normalized with $\mathrm{L}_{1}$ norm, generating a frequency vector

$$
\mathrm{H}^{\mathrm{X}}=\left[\mathrm{h}_{1}^{\mathrm{X}} \mathrm{h}_{2}^{\mathrm{X}} \ldots \ldots \mathrm{h}_{\mathrm{k}}^{\mathrm{X}}\right] \text {. }
$$

\section{Classification}

We use SVM with kernels as learning algorithm for the classifier. The kernels implicitly map the input space to a higher dimensional space[8,14].We used exponential kernel $\mathrm{X}^{2}$ for features, because it has been powerful discriminative tool for classification [14].

$k(\chi, y)=\exp \left(-y \sum_{i=1}^{N}\left(\frac{\left(\chi_{i}-y_{i}\right)^{2}}{\left[\left(\chi_{i}+y_{i}\right)\right]}\right)\right)$
With our features, the classification accuracy was high; especially they can model the classes.

\section{v. Experiments}

We test the proposed method on spine MRI images, a set of two types of spine MRI images. This collection contains more than 1000 spine MRI images, provided by the web site of spine health [9] and classified in two categories normal and abnormal. Experiments demonstrate our method's accuracy of classification and flexibility for a large scale spine MRI images. Multiple GLCMs produced from the two by sets specifying an array of offsets to the program.

These offsets define pixel relationships of varying directions (horizontal, vertical, and two diagonals) and 30 distances for each direction. In this case, each type of spine MRI image is represented by 30 GLCMs, and then four statistical features (Contrast, Correlation, Homogeneity and Energy) calculated from these GLCMs, and take the average for each feature in four directions for the distance 30, see Tables I and II. To facilitate the comparison between features of normal tissue and abnormal tissue, each feature plotted versus offset. The initial step of our method is to extract the local features using the method we have proposed that combines between cooccurrence texture features with bag of visual words to improve classification accuracy [10, 11-13].

In fact, to detect the contribution of our model using classification of spine MRI images, we introduce table II, which outline acquired values for average Haralick features. We note that our method significantly differentiate of two types of spine MRI images. These inspections are committed by the figures shown in Fig. 3-6 and table III. 
Proc. of the Intl. Conference on Advances in Information Processing and Communication Technology - IPCT 2016. Copyright (C) Institute of Research Engineers and Doctors. All rights reserved.

ISBN: 978-1-63248-099-6 doi: 10.15224/ 978-1-63248-099-6-60

TABLE II The average of Haralick features (Contrast, Correlation, Energy and Homogeneity) in four directions $\left(0^{\circ}, 45^{\circ}, 90^{\circ}\right.$, $135^{\circ}$ ) for normal and abnormal tissues in a set of 30 distances for spine MRI images.

\begin{tabular}{|c|c|c|c|c|c|c|c|c|}
\hline \multirow{2}{*}{$\begin{array}{l}\text { off } \\
\text { set } \\
\text { No. }\end{array}$} & \multicolumn{4}{|c|}{ Normal image } & \multicolumn{4}{|c|}{ bnormal image } \\
\hline & Contrast & Corr. & Energy & Homogeneity & Contrast & Corr. & Energy & Homogeneity \\
\hline 1 & 1.5056 & 2.96170 & 0.7091 & 2.8995 & 0.1003 & 2.3949 & 2.9615 & 3.2131 \\
\hline 2 & 3.6340 & 2.5551 & 0.6253 & 2.7292 & 0.1654 & 1.8533 & 2.9239 & 3.1924 \\
\hline 3 & 5.4761 & 2.2048 & 0.5728 & 2.6180 & 0.2037 & 1.5455 & 2.8939 & 3.1771 \\
\hline 4 & 7.0166 & 1.9144 & 0.5311 & 2.5289 & 0.2538 & 1.1451 & 2.8663 & 3.1637 \\
\hline 5 & 8.1809 & 1.6975 & 0.4978 & 2.4594 & 0.2919 & 0.8503 & 2.8442 & 3.1530 \\
\hline 6 & 8.9072 & 1.5653 & 0.4715 & 2.4050 & 0.3090 & 0.7337 & 2.8336 & 3.1479 \\
\hline 7 & 9.3160 & 1.4936 & 0.4505 & 2.3596 & 0.3180 & 0.6812 & 2.8277 & 3.1452 \\
\hline 8 & 9.5833 & 1.4490 & 0.4328 & 2.3173 & 0.3239 & 0.6553 & 2.8238 & 3.1435 \\
\hline 9 & 9.7802 & 1.4181 & 0.4180 & 2.2797 & 0.3289 & 0.6375 & 2.8199 & 3.1422 \\
\hline 10 & 9.9460 & 1.3932 & 0.4059 & 2.2473 & 0.3301 & 0.6517 & 2.8182 & 3.1415 \\
\hline 11 & 10.1818 & 1.3564 & 0.3973 & 2.2226 & 0.3306 & 0.6680 & 2.8164 & 3.1409 \\
\hline 12 & 10.5242 & 1.2999 & 0.3891 & 2.1939 & 0.3328 & 0.6686 & 2.8136 & 3.1402 \\
\hline 13 & 10.9225 & 1.2337 & 0.3810 & 2.1616 & 0.3359 & 0.6613 & 2.8106 & 3.1396 \\
\hline 14 & 11.3307 & 1.1666 & 0.3744 & 2.1295 & 0.3405 & 0.6380 & 2.8070 & 3.1388 \\
\hline 15 & 11.7091 & 1.1048 & 0.3687 & 2.0977 & 0.3474 & 0.5940 & 2.8012 & 3.1367 \\
\hline 16 & 12.0555 & 1.0495 & 0.3629 & 2.0670 & 0.3515 & 0.5790 & 2.7974 & 3.1355 \\
\hline 17 & 12.4400 & 0.9875 & 0.3583 & 2.0343 & 0.3576 & 0.5526 & 2.7918 & 3.1334 \\
\hline 18 & 12.8460 & 0.9223 & 0.3553 & 2.0034 & 0.3657 & 0.5112 & 2.7858 & 3.1310 \\
\hline 19 & 13.2030 & 0.8659 & 0.3525 & 1.9762 & 0.3701 & 0.4927 & 2.7830 & 3.1300 \\
\hline 20 & 13.5212 & 0.8162 & 0.3488 & 1.9495 & 0.3684 & 0.4818 & 2.7855 & 3.1304 \\
\hline 21 & 13.7613 & 0.7773 & 0.3463 & 1.9255 & 0.3571 & 0.4738 & 2.7984 & 3.1339 \\
\hline 22 & 13.9151 & 0.7506 & 0.3426 & 1.9092 & 0.3387 & 0.4663 & 2.8193 & 3.1399 \\
\hline 23 & 13.9724 & 0.7300 & 0.3386 & 1.8980 & 0.3164 & 0.4713 & 2.8516 & 3.1490 \\
\hline 24 & 14.0282 & 0.7005 & 0.3349 & 1.8875 & 0.2998 & 0.4674 & 2.8772 & 3.1560 \\
\hline 25 & 14.0915 & 0.6597 & 0.3315 & 1.8808 & 0.28054 & 0.46060 & 2.89903 & 3.16196 \\
\hline 26 & 14.1482 & 0.6030 & 0.3279 & 1.8703 & 0.2478 & 0.4586 & 2.9193 & 3.1679 \\
\hline 27 & 14.2802 & 0.5282 & 0.3245 & 1.8609 & 0.2313 & 0.4684 & 2.9352 & 3.1723 \\
\hline 28 & 14.3780 & 0.4522 & 0.3214 & 1.8594 & 0.2320 & 0.4651 & 2.9343 & 3.1723 \\
\hline 29 & 14.4122 & 0.3778 & 0.3199 & 1.8552 & 0.2339 & 0.4546 & 2.9315 & 3.1713 \\
\hline 30 & 14.3821 & 0.3057 & 0.3187 & 1.8558 & 0.2374 & 0.4415 & 2.9284 & 3.1701 \\
\hline
\end{tabular}

$\begin{array}{lllllll}\text { TABEL } & \text { III. } & \text { VERAGE } & \text { ACCURACY } & \text { OF } & \text { TWO } & \text { TYPES }\end{array}$ SPINE MRI IMAGES

\begin{tabular}{|l|l|}
\hline $\begin{array}{l}\text { Type of spine MRI } \\
\text { image }\end{array}$ & MAP \\
\hline Normality & 0.8321 \\
\hline Abnormalities & 0.3446 \\
\hline
\end{tabular}

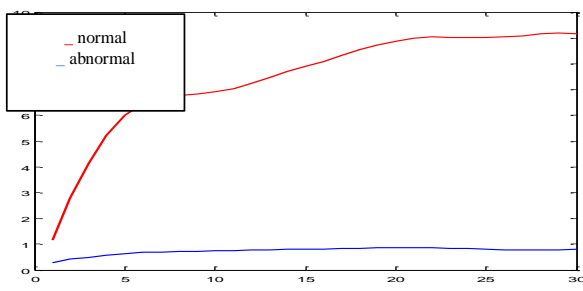

Figure 3. The average of statistical Contrast features From normal and abnormal spine MRI images

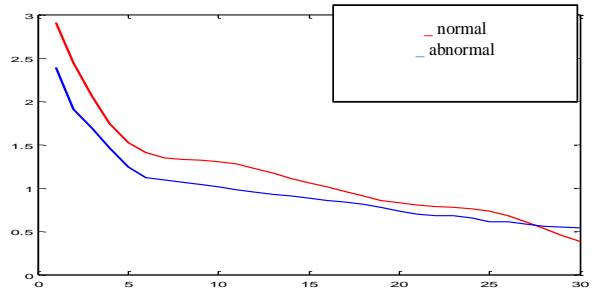

Figure 4: the average of statistical Correlation features from normal and abnormal spine MRI images

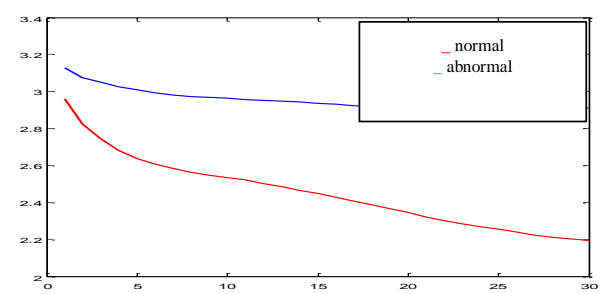

Figure 5: the average of statistical Homogeneity features from normal and abnormal spine MRI images

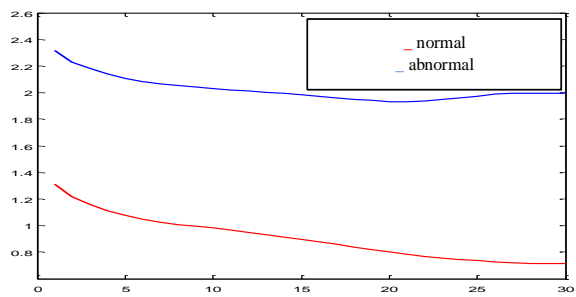


Figure 6: the average of statistical Energy features from normal and abnormal spine MRI images

\section{Conclusion}

This paper has addressed the problem of classification of normality and abnormalities of spine MRI images based bag of visual words, which are extracting gray level co-occurrence Haralick texture features like contrast, correlation, homogeneity and Energy, as words to develop discriminating power of bag of features based medical image classification. Our results indicate that more efficient feature for differentiating between normal and cancer is contrast followed by homogeneous and Energy features but correlation feature is not appropriate to distinguish between spine MRI images. Experiments demonstrate our method's accuracy of classification and flexibility for a large scale spine MRI images. In future work, we investigate of efficient algorithm to detect local features of various large scales of medical images.

\section{References}

[1] Faleh H. Mahmood, Wafaa A. Abbas,'Texture Features Analysis using Gray Level Co-occurrence Matrix for Abnormality Detection in Chest CT Images", Iraqi Journal of Science, 2016. Vol. 57, No.1A, pp: 279-288

[2] R.M. Haralick, K. Shanmungam, and I. Dinstein, "Textural Features of Image Classification," IEEE, vol. 3, pp. 610-621, 1973.

[3] Zhi, Li-Jia ; Zhang, Shao-Min; Zhao, Da-Zhe; Zhao, Hong; Lin, Shu-Kuan. Medical image retrieval using SIFT feature. Proceedings of the 2009 2nd International Congress on Image and Signal Processing,CISP’09, 2009.

[4] Saad Al-Momen, Loay E. George and Raid K. Naji.2015.'Texture classification using spline, wavelet decomposition and fractal dimension", Applied and Computational Mathematics, science publishing group,4(1):5-10.

[5] S.H. Raza, R.M. Parry, R.A. Mo, A.N. Young, M.D. Wang. "An Analysis of Scale and Rotation Invariance in the Bag-ofFeatures Method for Histopathological Image Classification". Medical Image Computing andComputer-Assisted Intervention - MICCAI 2011, vol. 6893, p. 66-74,2011.

[6] Avni, U; Greenspan, H; Sharon, M, et al. X-Ray image categorization and retrieval using patch-based visualwords representation. 2009 IEEE International symposium on biomedical imaging: fromNano to macro, vols. 1 and 2 pages: 350-353 Published: 2009.

[7] Dalia N. Abdul Wadood. 2014. Detection and Localization of Non-Melanoma Skin Cancer Using Texture Analysis , M.Sc. Thesis, Department of Computer Science, College of Science, Baghdad University, Iraq..
[8] Zayed N. and Elnemr, H. A. 2015. Statistical Analysis of Haralick Texture Features to Discriminate Lung Abnormalities, International Journal of Biomedical Imaging, 2015, Article ID 267807, pp:1-7.

[9] MRI scan of spine, spine health, http://www.spine-health.com

[10] Riadh B., Abir M. and Jalel A. , "Using a Bag of Words for Automatic Medical Image Annotation With a Latent Semantic", International Journal of Artificial Intelligence \& Applications (IJAIA), Vol 4, No.3, May 2013

[11] Ahmed M., Abdullah A., Bassem Z,, M. Awedh, " medical image classification using multivocabulary", Asian journal of applied research , 8(1), 2015.

[12] Asavari G. J., A. S. Deshpande,"Review of Face Recognition Techniques", International Journal of Advanced Research in Computer Science and Software Engineering, Volume 5, Issue 1, January 2015

[13] Khawlah Hussein A. 1, T. Wang, "On the Development Robust and Fast Algorithm of Action and Identity Recognition", American Journal of Networks and Communications; 4(5), 2015.

[14] Khawla Hussein A., Entesar B,. Nadra J, "Texture Features Based Bag of Visual Words for a Spine MRI Images", Proc. of the Fourth Intl. Conf. Advances in Computing, Communication and Information Technology- CCIT 2016. ISBN: 978-1-63248-092-7, USA.

\section{About Authors}

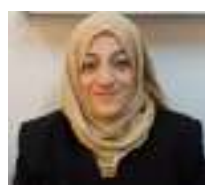

Khawlah H.Ali received M.Sc. degree from the University of Basrah in 2001 and the Ph.D degree from Huazhong University of Science and Technology (HUST), Wuhan, China in 2015. She is author of many published papers in international journals and conferences in different topics and her current interests include computer vision and pattern recognition, image processing, machine learning and security applications in computer fields.

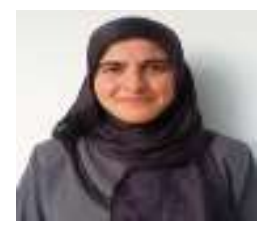

Entesar Barges Talal finished the BSc. in the University of Basrah, college of education, Dept.of computer science at 2006.The M.Sc. has been finished in the University of Basrah, college of science, Dept. of computer science at 2015 with specialization biometric and pattern recognition. interest with many fields like machine learning, computer vision, Artificial intelligent, web design and semantic web. The languages programs that she supports Pascal, $\mathrm{C}++$, prolog, visual basic, php, HTML, java script, Matlab. 